\title{
TRANSPORT IN SMALI AND/OR RANDOM SYSTEMS
}

\author{
PROGRESS REPORT
}

For the Period June, 1986 to May, 1987

Prepared for

THE U. S. DEPARTMENT OF ENERGY

DE-FG02-84 ER45058

Prepared by:

Professor Melvin Lax

Distinguished Professor

Principal Investigator

Department of physics

The City College of CUNY

Convent Avenue e $138 \mathrm{th}$ street

New York, NY 1.0031
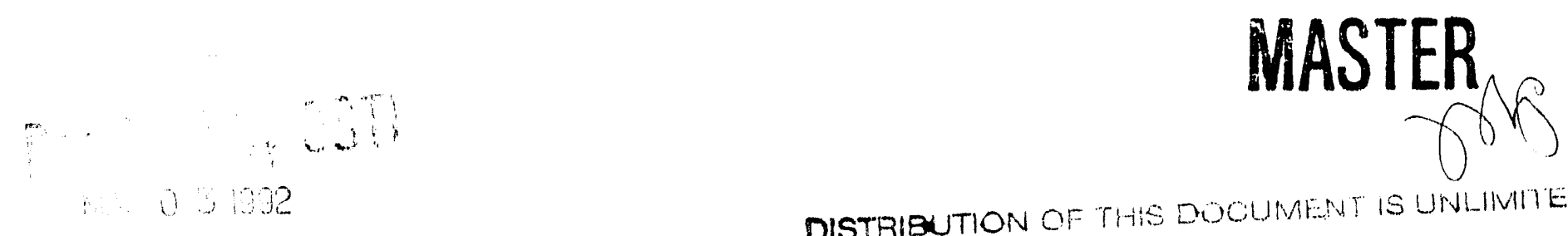

DISTRIBUTION OF THAS DOCUMENT IS UNLLIVITED 
TRANSPORT DN SMALL AND/OR RANDOM SYSTEMS

\title{
Principal Investigator Melvin Lax
}

City College of New York, New York, NY. 10031

\section{DISCLAIMER}

\begin{abstract}
This report was prepared as an account of work sponsored by an agency of the United States Government. Neither the United States Government nor any agency thereof, nor any of their employees, makes any warranty, express or implied, or assumes any legal liability or responsi. bility for the accuracy, completeness, or usefulness of any information, apparatus, product, or process disclosed, or represents that its use would not infringe privately owned rights. Reference herein to any specific commercial product, process, or service by trade name, trademark, manufacturer, or otherwise does not necessarily constitute or imply its endorsement, recom. mendation, or favoring by the United States Government or any agency thereof. The views and opinions of authors expressed herein do not necessarily state or reflect those of the United States Government or any agency therenf.
\end{abstract}

\section{PERSONNEI.}

The following people bave received at least parial support from this grant during the last three years

\section{Research Aseociatea}

Wei Cai

Shirish M Chitanvis (Now at Lom Alamos Nhtioanal Laboratory)

Ya-Qiu Jin

M Cristina Marchetti (Now at the University of Illinois at Chicago)

Chui Lin Wang (Previously a student) 
- 2 -

?

Student=

Boris Yudanin

Ting-Fang Zheng 


\section{DNTRODUCTION}

\section{(a) Technological Importance}

The introduction of molecular beam epitary ${ }^{1}$ (MBE) and metalo-organic chemical vapor deposition $^{2}$ (MOCVD) has permitted the engineering of heterostructures from multilayer cases to single quantum wells. The additional procedure of modulation doping introduced by Stormer, Dingle, Gow sard and Wiegmann has permitted mobilities of the order of $10^{6} \mathrm{~cm}^{2} / V$ sec to be attained. "Band structure engineering" has also enhanced the posibilities for construction of semi-conductor lacers.

A large number of devices based on these techniques has caused the physica of film heterostructures to be a field of intense study."

Among the topics of interes to us are bot electron effects, electron-phonon interactions and resonant tunneling. As the dimension of devices shrink, ordinary voltages lead to strong fields and hot electrons. They also lead to devices whase dimension is not much larger than the mean free path. Thus ballistic, or quasi-ballistic transport becomes important

\section{(b) Transport in Small Systems}

We have recently explored the trasport of phonons in GaAs at sufficiently low temperatures that the slab thickness is just several mean free paths ${ }^{5}$ Our Vonte Carlo results, in the regime in which down-conversion can be aeglected, have led to sufficient insight to lead to a useful, approximate azalytic model. These results should be of use in other problems as the transition is made from diffusive to ballistic transport A copy of a preliminary report is enclosed.

\section{(c) Quani - 2D Systems}

Electron-phonon interactions in a single quantum well, or in a heterostructure can, 20 a first approzination be regarded as an interaction between a two-dimensional (2D) electron and threedimensional (3D) phonons. This remark also applies to electrons (or holes) in inversion layers or MOS structures. Peter Price recognized that physical answers in such a situation depend on the effective number of phonon modes that coupled in, and that this number appears to depend on the arbitrary choice of quantization volume

Cai, Marchetti and $P$ have show $n$ that this problem can be solved by coupling the electrons to wave-packets of phonons that are localized in the vicinity of the quantum well. But what shape 
should be chowen for the phonon packets? A method Bogolyubov developed years ago for dealing with transport in fluids was employed This method ivtroduces a small number of "macroscopic" variables and adiabatically eliminates the remaining varialbles. The result is a reduced transport equation for the slow variablea.

An important physical decision must be inade as to what the appropriate "macroucopic" variables are. The choice we made for the slow variables $w$ as the reduced one-body density matrices of both the electron and phonon gas The beauty of this choice is that it permits one to retain offdiagonal elements in $q_{2}$ and $q_{2}^{\prime}$, the components of wave vector perpendicular to the well walle Thus no choice has been made for the shape of the wave packets

Because the kernel of the integral equation for the phonon density matrix is found to be a product (more precisely a sum of four products) the natural procedure for solution of such an integral equation leads to the phonon packet representation. Moreover, there is one choice of packet for each quaptum well state. Thus at low temperatures in which only a small number of well states are occupied, the number of equations is equal to the number of well states occupied This good fortune means that the coupled integro-differential equations are reduced to a trivial number of coupled ordinary differential equatione In this way, a rigorous approach to the paradox raised by Prici also leads to a practical method of solution.

Having broken the Gordizn knot of the proper method of coupling 2D electrons to 3D phonons, the solution of many problems of intereat are possible. We have alreacty reported, in the above paper, on non-equilibrium electron-phonon scattering in heterojunctions Because the electrons coupled into individual phonon packets the equilibrium of these packets is appreciably disturbed, a back-reaction sets up, and the steady state energy los rate of a hot electron can be greatly reduced A second calculation by Cai, Mrehetti and Las solves the associated problem of time-dependent electron relaration. Additional problems involve a strong electric field, multi-subband sccupation and transport in the presence of a magnetic field.

The work on quasi 2-D systems will be the subject of an invited paper at the nert international conference on Hot Electrons in Semiconductors

\section{(d) Noise In Small Systems}

Because noise may be an imporant factor in the limitu of achievement of small systems including heterostructure and semiconductor lasers, we purpose to consider the development of tools to calculate this noise Although we already have an arsenal of tools on clasical noisen-14 and 
quantum noise, ${ }^{15-27}$ we expect that new techniques must be developed because of the quasi-2D nature of some systems, and quantim nature of the motion perpendicular to the beterostructure walls.

We have already seen that transport in electron-phonon systems in non-equilibrium require the use of density matrices and appropriately determined phonon wave-packeta. The same will clearly be true for noise in such systems.

\section{(e) Laser Propagation in the Atmoophere}

The random probleme arocinted with propagation through a random medium containing particulate scatterers including aerosols are well-known, but the emphasis has been on solutions for an incident plane wave. The problem of dealing with an incident Gaussian beam, initially narrow in both angle and transverse dimension has only recently been attempted. See Zardecki and Gersth Chitanvis ${ }^{29}$ The need for a comprebensive treatment is clear.

\section{(f) High Intensity Laser-Aerosol Interactions}

When a laser beam has high intensity, its interaction with an aerosol becomes nonlinear. Shocks and rarefaction waves can form at high powers. Evaporation will be important at lower powers and longer times Such problems require a numerical treatment on a large computer because of their hyperbolic character and dependence of two space and one time variable However, solution of special cases eq. of planar or spherical symmetry to provide tests of the available 2D hydrocodea are desirable.

We propose to make ure of CAVEAT, ${ }^{30}$ recently written $2 \mathrm{D}$ bycrocode involving a halfdozen authors over several years at Los Alamoe A strong point of this code is that it permite the handling of shocks by treating each cell side as if it wete a potential discontinuity boundary. It then applies Godonov's procedure treating each discontinuity as if it involved an infinite place and usea the Riemano scaled solution of the latter problem.

I have questions concerning the accuracy with which the code accurately reflects Godonov's original ideas (as opposed to his original algorithm) Also Chitanvis has suggested, and I agree that the scaling assumption in the Rieman solver is no longer valid when thermal energy is released into the system. The problem, therefore, is to see if corrections incorpornting the new physica can be incor porated into the code without changes chat would disturb its otherwire excellent featurea. 


\section{(g) Reflection From Random Surfacea}

An improved theory of scattering from rough random surfaces will be attempted that includes correlation effects on the surface and multiple scattering.

Somew hat more detailed dexcription of the previous success, and proposed new work on the topics mentioned above, are included in the sections written below by my students and research associates. A detailed discussion is given below.

\section{TRANSPORT PROPERTIES OF CARRIERS IN SEMACONDUCTOR QUANTUM WELL (CAI and Zheng)}

\section{Nonequilibrium phonon effect on hot carrier transport}

Recent exeriments have shown that the presence of nonequilibrium optical phonons induces important effects on hot carrier properties in semiconductor quantum wells. We have proposed a description of hot phonon dynamics in quantum-well structures that properly treats the coupling of 2D - carriers and 3D lattice excitations?. Our approach rests on the derivation of a phonon kinetic equation that applies in the presence of the strong spatial inhomogeneity due to carrier confinement and on the introduction of a "phonon wave packet " representation. We used this approach to evaluate the effect of nonequilibrium optical phonons on relaxation of hot electrons in heterojunctions bock in a steady state ${ }^{7}$ and in a time dependent situation ${ }^{8}$. A reduction of the electron cooling rate of a factor 6-8 was obtained in the steady state case. The suppression of tot electron cooling is even stronger in the time dependent case. Both results are in agreement with recent experiments.

Since the presence of nonequilibrium optical phonons can greatly affect the distribution function of electrons in their relaxation procese, various electron properties are changed due to this effect. Previous theoretical results, mort of which are calculated based on the assumption that phonons art: in equilibrium with heat bath, should be reexamined.

Recently we are studying the effect of nonequilibrius optical phonons on hot electron transport in heterojunctions in the presence of a uniform extermal strong electric field $\mathbf{E}$. Scatterinis with remote impurities and acourtic phonons via both deformation potential and piezoelectric interaction are included. The decay of the nonequilibrium LO phonons is described by a single relaration 
time Our initial results show that there are important effects on the electron transport due to presence of hot phonom We find that reabeorbtion of nonequilibrium optical phonons increanes the weak field-lectron mobility. At high field the presence of nonequilibrium optical phonons enhances electron temperature much farter with the field than that in the case of equilibrium phonons and the mobility normalized to its weak field value decreasea faster with the field than obtained when the LO phonons are in equilibrium. The occupation number of nonequilibrium LO phonons is strongly anisotropic in the direction of electric field. We also study the time dependent evolution proces after applying an electric field. We find the time needed for approach to the steady state in the presence of hot phonons becomes much longer than that in the equilibrium phonon case ${ }^{31}$

A drifted temperature model and a single subband approximation, however, are used in our recent calculation. A more detail computation, which includes multi-aubbands and multi-valleys is needed, especially, for the case that a high electric field is applied A calculation of cooling rate of hot electrons in the case where the multi-subbands are occupied has been worked out ${ }^{32}$ which shows that our deacription of "phonon wavepacket" can be used in multi-subbands case without difficulty.

Recently, we are also studying the nonequilibrium phonon effect on transport of holes in quantum wells.

\section{Transport of an electron-bole gas in a quantum well}

Recently there has been much interess in the $2 \mathrm{D}$ semiconductor system where the electrons and holes coexist. The experiments have shown that when a strong electric field is applied to this system, the electrons and the boles have different drift velocities and different temperaturean ${ }^{13}$ Under some special conditions it has been discovered that the electrons can move along positive direction of the external electric field ${ }^{34}$ Nhmely, an abwolute negative mobility of electrons is shown in addition to the scattering of electrons and holes with phonons and impurities, the electron-bole scattering plays a important role in the transport of this system, which produces the "electron-hole drag" effect

We address to the transport phenomena in this system. the dynamic scieening effect in this system has a special form since electron-hole interaction is as important as electron-electron ( bolehole) interaction. Also this streening effect make the coupling of electron-phonon (impurity) interaction with hole-phonon (impurity) interaction. The subband wavefunctions of electrons and holes in this system are different from the case that only electrons (or holes) exish because the common selfconsistent potential is a superposition contributed from both electrons and holea To our know ledgen these have not been carefully considered before. We have also derived a set of coupling equations to obtain temperatures and mobilities for both electrons and holen. The conditions of the absolute 
$-8-$

4

negative mobility of electrons we obtained is different from what shown in previous paper by other authors. ${ }^{34}$ The riumerical computation is in progreas 


\section{WORK NEAR COMPLETION}

\section{(1). Scattering from correlated points of a random rough surf ace}

EM wave scattering from a random rough surface arises in many areas of science and engineering, such as atomic scattering from a solid surface, and remote sensing for Earth terrain. When the correlation leagih and variance of roughness are much larger than the wavelength, the Kirchhoff approximation is applied. The variables of the roughoess heights and slopes of the specular points, have previously been dealt witb as independent Therefore, the contribution from such corre lation was totally omitted In the high frequency limit, the scattered intensity finally is only a summation from independent stationary points

However, our study elucidates that for any pair of specular points in one Fresnel zone, as long as their distance is not larger than the correlation length (note that the latter might be mucb larger than the wavelength), the roughness heights and slopes are correlated. Vote that most contribution to the scattered intensity happen to come from this small region. The contributions from these correlations are appreciable, and should be taken into account for computation of the scattered intensity. A general approach and analytic formulation to take account of the correlation have been developed. The difference caused by the correlation is discussed, and numerical results are also provided in the manuscript.

\section{(2). Multiple scattering from a randomly rough surface}

Solution for scattering from a randomly rough surface has been achieved by the well-know n methods of Kirchhoff approximation, small perturtation theory ${ }^{47,48}$, and recently by the full wave ${ }^{49}$ approach, and others. However, these approaches geserally are restricted to the sungle scaturing approximation. Multiple interactions of a scattered wave aith the surface were distegarded. The multiple scattering theory for a randomly rough surface (mostly, for a perfect conducting surface) has been discussid by using a diagrammatic method, so Stochastic Fourier transformation, smoothing $^{51.52}$ method, etc. However, these discussions are largely formal, and the emphasis is on the anslytic structure of the integral equation of scattered field. These methods do not seem to be tractable enough yet to quantitatively assess the effect of multiple scattering.

We $w$ ish is to develop an explicit formula to take multiple scattering into account. The polarized scattered power from a randomly rough surface is contributed to not only by single scatcered waves, but also by those high order scattered waves which undergo re-incident and re-bounced 
scatterings in various directions. The multiple scattered field is represented as a Born series By using the stationary phase method, the scattered field with the single scattering assumption has been freviously obtained. For the double scattering, the scattered power is contributed by those statistically distributed pairs of the stationary points, which are illuminated, not obscured, and there is no obstacle between them. Shadowing functions are obtained by making use of the method of random variable overshoots to the determinant ray. The polarized scattered fields for double scattering can be expressed by the sequential-scatterings ( second-order Born approximation) and shadowing factors. Then, an explicit formula for the bistatic double scattering intensity can be statistically obcained, and by iteration the higher order scattering can be estimated. Thus, the contribution to the polarized scattered power from high order scattering from a randomly rough surface with given statistics can be evaluated. Functional dependence of the scattering and numerical calculations are obtained, and discussed in the manuscripe.

\section{LIGHT TRANSMISSION DN A PARTICULATE MEDIUM (B.Yudanin)}

Light propagation through an optically thick particulate medium is basically a mulufle scattering problem in which rays or photons traverse a medium of scatuerers and undergo many scattering events before escaping. A natural framework to deal with this type of problem is provided by the theory of radiative transfer. The linear Boltzman equatiun - in the context of radiatuve energy also termed the equation of transfer - governs the radiatun field in a medium that absorbs emits, and scatters radiation.

The complexity of the equation of transfer forces one to implement numerical methods of solution. Lnfortunately, such calculations become a rather formidable task even on the most pourerful supercomputers available today.

Due to the numerical complexities, an important aspect of transpmet theory is volves development of simpler approximate descriptions. The well-known small-angle approximation procedure provides an example of the algorithm applicable to scattering from particles with highly anisotropic phase function. When the particle density is sufficiently tenuous, a single-scattering approumation is adequate. When the scattering is nearly isotropic, a diffusion approximation becomes adequate even in the presence of absorption. With moderate anisotropy, the quantitative measure of which is the mean cosine of the scattering angle, the diffusion approximation gives relatively simple and good solutions when the particle volume density is high. Of course, the diffusion equation will contain the necessary absorptive term.

One way to simplify the transport equation is to reduce it to a form of three simultaneous 
partial differential equations for the reduced field amplitude, the forward-and backscatlered uradsances. The scattering and diffusion coefficients it these equations will be defined w terms of Mie functions. The determination of those coefficients will be one of the main aspects of our invesugation. The theory which we are planning to develop will be based on multiple-scattering theory.

As a starting point in our investigation we consider the correlation function of the electric field of the radiation of frequency $\omega$ and velocity $c$.

$$
\Gamma(R, r, \Omega, \omega)=\overline{E\left(R+\frac{r}{2} ; \Omega+\frac{\omega}{2}\right) E^{2}\left(R-\frac{r}{2} ; \Omega-\frac{\omega}{2}\right)}
$$

The Fourier transform of $\Gamma$ with respect to $r$ and $\omega$ is proportional to the intentity of radiation at $R$ with wave vector $q$. This quantity can be expressed in terms of the average of the product of two Green's functions.

To get a generalized transpor equation for the intensity of the radiation in the presence of randomly distributed scatterers we will first obrain an integral equation for the correlation function $\Gamma$ in the approximation which is consistent with a CPA for the average feld E.

First we introduce the coherent dielectric constant $\epsilon_{c}$ and the (herent Green's funcuca $G_{c}$. such that there will be no scattering on the average from such a media le.g, the average $T$ - matrix is zero). This will lead to the condition that the average Green's functurn of the field is equal to the coherent one. The coberent potential and the corresponding coherent Green's function can be found self consistently as was done in any standard C.P.A calculation for, say, Lquid - type disorder.

As stated above, to find intensity we need to know the average of the product of two Green's functions at different space-time poines. An equation, consistent with the C.P.A for the average of the product of two Green's functions will contain as a kernel an average of the product of the $t$ -matrices for the scatterer, imhedded in:o otherw ise coherent medium

After some manipulations arrive into a familiar form of a differential transport equation:

$$
\begin{aligned}
& i\left[\omega \frac{\partial}{\partial t}+\mathbf{q} \cdot \nabla\right] \Gamma^{n n c}(\mathbf{R}, \mathbf{q}, t)+\frac{1}{2} \int d \mathbf{R}^{\prime} \Delta \nu_{c}^{\prime}(\mathbf{R}-\mathbf{R} ; \mathbf{q}) \Gamma^{n c}(\mathbf{R} \cdot, \mathbf{q}, t)
\end{aligned}
$$

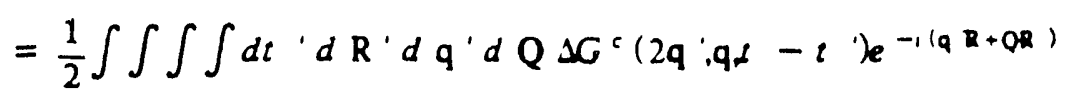

$$
\begin{aligned}
& \times t\left(\mathbf{q}+\mathbf{q}^{\prime} ;-\frac{\mathbf{Q}}{2}-\mathbf{q}^{\prime}\right) t\left(\mathbf{q}-\mathbf{q}: \frac{\mathbf{Q}}{2}-\mathbf{q}\right) \Gamma\left(\mathbf{R}: \mathbf{q}: t^{\prime}\right)
\end{aligned}
$$

To get a set of equations for the intensities and amplitudes of the form of the diffusion and the small 
angle transport, we will decompose the $t$-matrix for the scatterer into a small angle part and the uniform (large angle) part which will be separately responsible for the arising paraxial and duffusion equations for the transport. It seems to be reasonable to incorporate the wide angle scatiering into a new "coherent medium", and to keep the small angle part of the scattering in the kernel of the equation (2).

In our following work we plan to simplify this full transport equation to the form of coupled equations for the small angle and for the diffusion scattering. We also plan to get a numerical solution of that system of equations.

\section{LASER GENERATION OF SHOCK WAVES DN DROPLETS (C. L Wang)}

The generation of shock waves in droplets by laser beam illumination has been studied in recent years. The droplets are first heated up by irradiation of laser beams, then expand and explode. producing shock waves and rarefaction waves. The problem is involved in two aspects, electrodynamic and hydrodynamic. The first aspect is to consider the interaction between electromagnetic waves and small droplets which causes the energy transfer from laser beams to water droplets. This procedure is governed by laxwell equations. The second aspect is it the investigate hydrodynamical response which is determined by a set of conservation equations (m.Ls, momentum and energy). The two set of equations are strongly coupled through source terms in the primitive equations and through the constitutive relations.

The feedback amongst the various electromagnetic and hydrodynamical equations can give rise to very unusual behavior. In fach in computer sumulations one might be hard pressed to distinguish the true physical behavior from numerical rubbish. However, the two very different aspects of the problem suggest that much can be learned by attacking them separately at the star. For exampie, the byarodynamic equations can be solved assuming the aboorption of laser radiation is know n.

We propose to emphasize first the hydrodynamical aspects of this problem. To a first approximation, the effect of EITR on a small droplet is to generate heat withun the droplet due to aboorfuon. The rate at which the heat is generated determines the response of the droplet. The heating can vary over a wide range as determined by the complex index of refraction. the intensity of the radiation source, and the optical size of the droplet. The droplet shows an equally wide range of response, from slow evaporation to explosion.

A large body of literature has developed considering many aspects of this problem. For exam-

ple, the articles by Caris and Brock ${ }^{53}$, Lax and Yudanins4, Prishivalko ${ }^{55}$, and Chitanvis 50 illurrate 
the wide range of effects that are being considered. Most of the current work addressing a realistic simulation of the hydroctynamics bas dealt with the slow vaporization regime ( see for instance, Armstrong ${ }^{57.50}$ and Sageev 59 ).

We will work on the explosive vaporization regime of this problem. In this regime convective effects dominate diffusive effects. Convective effects are driven by an imbalance in pressure feld. Convection becomes important when the vapor pressure of the evaporating droplet becomes comparable to the pressure of the surrounding gas Linder these conditions, the rate of evaporation is greater than the rate at which diffusion can "conduct" mass away from the droplet. As the temperature and, therefore, the vapar pressure and the evaporation rate of the droplet rise, convection becomes an effective mechanism for the man transport away from the droplet At higher temperatures it becomes the dominant mechanism.

We propose to choose a simple but very dynamic test problem as a first step towards developing a general solution for the convection dominated regime. By chonsing a relatively simpie hydrodynamical system, we can concentrate on simulating the physical properties of the droplet realistically. This in turn will help us determine what other physics (such as nutleation and particle growth) might arise due to strong convective effects. The problem that we are planning to solve can be posted as follows a pure water droplet small compared with the wavelength of the incident light, is subject to a very shorh very intense pulse of laser radiation. The optical size of the droplet is in the Rayleigh limit, thus it absorbs energy uniformly. In one special case, the very short duration of the pulse can ensure that the heating process ends before any fluid motion occurs. This implies that the droplet heats at constant volume.

Heating the droplet at constant volume causes its pressure to increase enormously (as bigh as 3000 atmospheres). The high pressure causes the droplet to expand violently against the atmosphere. The strong dyuamies generate shock waves and associated shock heating in some regions, and adiabatic expansion, cooling, and the possibility of nucleation in otber regions. We plan to consider all these possibilities in our work.

A Lagrangian-Eulerian rezoning code. CAVEAT ${ }^{30}$, from Los Alamos is in the development stage which can numerically solve the equations of transient multimaterial, compressible fluid dynamics. This code employs the Godunov technique, which allows close coupling between cellcentered pressure and velocity felds and serves as the basis for handling jump disconnection between regions of fluid. The ingenious Godunov method has the virtue of treating any real discontinuity the fluid may contain more realistically than other known methods including the viscosity methods da because the methods eventually treat each mesh point as if it has a discontinuity. But the Godunov 


\section{$-14$}

:

method essentially makes use of the Riemann solver, which came from an analytically solvable onedimensional tube problem. The Riemano solver is based on a scaling assumption not valid in the presence of source terms. The applicability of the unmodified Godunov method must be examined. In the mean time, we try to do a parallel test. We plan to modify our existing code, which uses the POST package, to calculate a simple spherical problem with a uniformly heating source The results will be compared with the one gotten from CAVEAT. If the result came from the CAVEAT is not satisfied a modification to the Goctunov technique is certainly needed before making use of CAVEAT in the presence of strong thermal sources

\section{REFERENCES}

[1] A. Y. Cho, Thin Solid Film 100, 291, (1983)

[2] P. D. Dupois, Science 226, 623, (1984)

[3] See the review by H. L. Stormer, Surf. Sci 132, 519, (1983)

[4] See the review by V. Narayanamurti, Physics Today 37, 24, (1994)

[5] M Lax, V. Larayanamurti, R. C. Fulton and N Holzwarth, Mbnte Carlo Calculations of Phonon Transport, Phonon Scatcering in Condensed Vlatter V, 335-337, (1986)

[6] Peter Price, Physica 134B, 164, (1985)

[7] W. Cai, M. C. Marchetri and M. Lax, Nonequilibrium electron-phonon scattering in semiconductor beterojunctions, Physical Review B 34, 8573-8580, (1986)

[8] M Cai, M C. Marchetti and M Lax, Vonequilibrium Phonon effect os time-dependent re laxation of bot electrons in semiconductor heterojunctions, Physical Review B 35, 13691372. (1987)

[9] M Lax, Fluctuations from the Nonequilibrium Steady State, Revs Mbd Phys. 32, 25-64 (1960).

[10] M Lax and P. Mengerh Tolluence of Trapping, Diffusion and Recombination on Carrier Concentration Fluctuations," J. Phys. Chem. Solids 14, 248-267 (1960). 
[11] M Lax, "Classical Noise III. Vonlinear Markoff Processea," Revs Mod Phys. 38, 359-379 (1966)

[12] ML Lax, "Classical Noise IV: Langevin Nethods," Revs, Mod Phys. 38, 541-566 (1966)

[13] M Lax, "Classical Noise V: Woise in Self-Sustained Oxcillators," Phys. Rev. 160, 290-307 (1967).

[14] R.D. Hempstead and M Lax, "Classical Voise VI: Voise in Self-Sustained Oscillators Near Threshold," Phys Rev. 161, 350-366 (1967).

[15] M Lax, “Generalized Mbbility Theory," Pbys Rev. 109, 1921-1926 (1958).

[16] M Lax, "Formal Theory of Quantum Fluctuations from a Driven State" Phys Rev. 129. 2342-2348 (1963).

[17] M Lax, "Quantum Relaxation, The Shape of Lattice Absorption and Inelastic Neutron Scattering Lines," J. Phys. Chem Solids 25, 487-503 (1964).

[18] M. Lax, "Quantum Noise V': Phase . Voise in a Homogeneously Broadened NLaser," in Physics of Quantum Electronics, P. L Kelley, B. Lax and P. E Tanenwald, eds. (.MrGraw-Hill Book Co. Inc, NY. 1966) pp. 735-747.

[19] M Lax, "Quantum . Vise VII: The Race Equations and Amplitude Noise in Lasers," J. Quantum Electronics, QE-3, 37-46 (1967).

[20] H. Cheng and ML Lax, "Quantum Voise VTI: Harmonic Oscillator Relaxation from Definite Quantum States" in Quantum Theory of the Soiid State, P.-O. Lowdin, ed. (Academic Press, In:, NY. 1960) pp. $587-600$.

[21] M Lax and W. H. Louisell, Quantum . Vise R: Quantum Fokker-Planck Solution for Laser Noise," J. Quantum Electronica, QE-3, 47-58 (1967).

[22] M Lax, "Quantum Voise X: Density .Tatrux Treatment of Field and Population Difference Fluctuations," Phys Rev. 157, 213-231 (1967).

[23] M Lax, "Quantum Noise Xi: Multitime Correspondence Between Quantum and Classical Stochastic Processes," Phys. Rev. 172, 350-361 (1968). 
[24] M Lax, W. H. Louisell, "Quantum Noise XI: Density Operator Treatment of Field and Population Fluctuations" Phys. Rev. 185. 568-591 (1969).

[25] M Lax, "Quantum Noise Xm: Six Classical Variable Description of Quantum Laser Fluctuations" Phys. Rev. 172, 362-371 (1968).

[26] M Lax and M Zwanziger, "Exact Photocount Distributions for Lasers Vear Threshold," Phys. Rev. Letters, 24, 937-940 (1970).

[27] C. H. Henry, P. S. Henry and $M$ Lax, Partition Fluctuations in Vearly Single- LongitudinalMode Lasers," J. of Lightwave Technology LT-2, 209-210, (1984).

[28] A. Zardecki, S. A. W. Gersth - Multuple - Scattering Analysis of Laser Beam Propagation in the Atmosphere and Through Obscurants ", LA.l工 Reporh (1983).

[29] S.M Chitanvis, Proc of the 1986 Conference on Obscuration and Aerosol Research.

[30] F. L Addessio, Daniel E Carroli, John K. Dukowicz, Francis H. Harlow, James 2 J Johnson, Bryan A. Kashiwa, Mathew E lbitrud, Hans M Ruppel, CAVEAT: A Computer Cade for Fluid Dynamics Problems with Large Distortion and Internal Slif. Los Alamos Vational Laboratory Report LA-10613-..S (1986)

[31] W. Cai, M C. Marchetti, and M Lax, to be submitted to Phys. Rev. B

[32] M C. Marchetti and W. Cai, Hot Electron Relaxation in Quantum-Well Structures MultiSubband Occupation, (accepted for publication) Pbys Rev. B 35, .lay 15 (1987).

[33] R. A. Hopfel, J. Shah, and A. C. Gossard, Phys. Rev. Leth 56, 765 (1986).

[34] R. A. Hopfel, J. Shah, P. A. Wolff, and A. C. Gonsard, Phys. Rev. Leth 56, 2736 (1986).

[35] L Tsang and J. A. Kong, Radio Science, 14, 3, 359, (1979)

[36] M A. Zuriga and J. A. Kong, J. Appl. Phys. 51, 10, 5228, (1980)

[37] M. Lax, Multiple Scattering of Waves [. The Effective Field in Dense Systems Phys. Rev. 85, 621-629, (1952) 
[38] M Lax, Wave Propagation and Conductivity in Random Media, SIA.lF.IS Proceedings VI, (1973)

[39] L. Tsang and A. Ishimaru, J. Elect Waves \& AppL 1, 59, (1987)

[40] Y. Q. Jin, Ph.D. Thesis, 9. (1985)

[41] A. Zardecki, S.A.W. Gersth and J.F. Embury, A pplied Optics, 22, 1346, (1983)

[42] A. Zardecki, S.A.W. Gersth and R.E Dekinder, Jr, A pplied Optics, 25, 3508, (1986)

[43] B. W. Fow ler and C. C. Sung, Applied Optics 17, 1797, (1978)

[44] K. Shimizu, JOSA A 73, 504, (1983)

[45] J. E. Gordon, JOSA A 2. 156, (1985)

[46] G. Stephens, J. Quant. Spectrosc. Radiat. Transfer 36, 51, (1986)

[47] P. Beckmann and A. Spizzichino, Pergamon Press, . YY, (1963)

[48] F. G. Bass and I. .M Fuks, Pergamon Press, .1Y, (1979)

[49] E Bahar, Radio Science 16, 1327, (1981)

[50] J.A. DeSanto, J. Tlath. Ptys 27, 377, 1986

[51] G. S. Brow n, IEEE Trans. Anl Prop. Ap-32, 1308, 1984

[52] G. S. Brown, IEEE Trans. Anc Prop. Ap-33, 48, 1985

[53] J. C. Caris, J. Brock. “ Explosion of a Water Droplet by Pulsed Laser Heaung ". Linpublished. (1986).

[54] M Lax, B. Yudanin, In "Proceedings of the 1985 CRDC Scierutic Conference on Obscuration and Aerosol Research ", (1986).

[55] A. P. Prishivalka * Water Droplets Irradiated by a Pulsed Laser ". Sov. Phys. 26, 149, (1985) 


$$
\frac{-18-}{4}
$$

[56] S. M. Chitanvis “ High Energy Laser Lnteractions with Water Droplets ", AppL Opt 24, 35.52 (1985).

[57] R. L Armstrong, “ Aerosol Heating and Vaporisation by Pulsed Light Beams ", Appl Opr 23 148, (1984).

[58] R. L Armstrong, "Interaction of A bsorbing Aerosols with Lntense Light Beams ". J. AppL Phys. 56, 2142, (1984).

[59] G. Sageev, “Laser Heating of an Aqueous Aerosol Particle ”, Appl. Opz 23, 4368, (1984). 

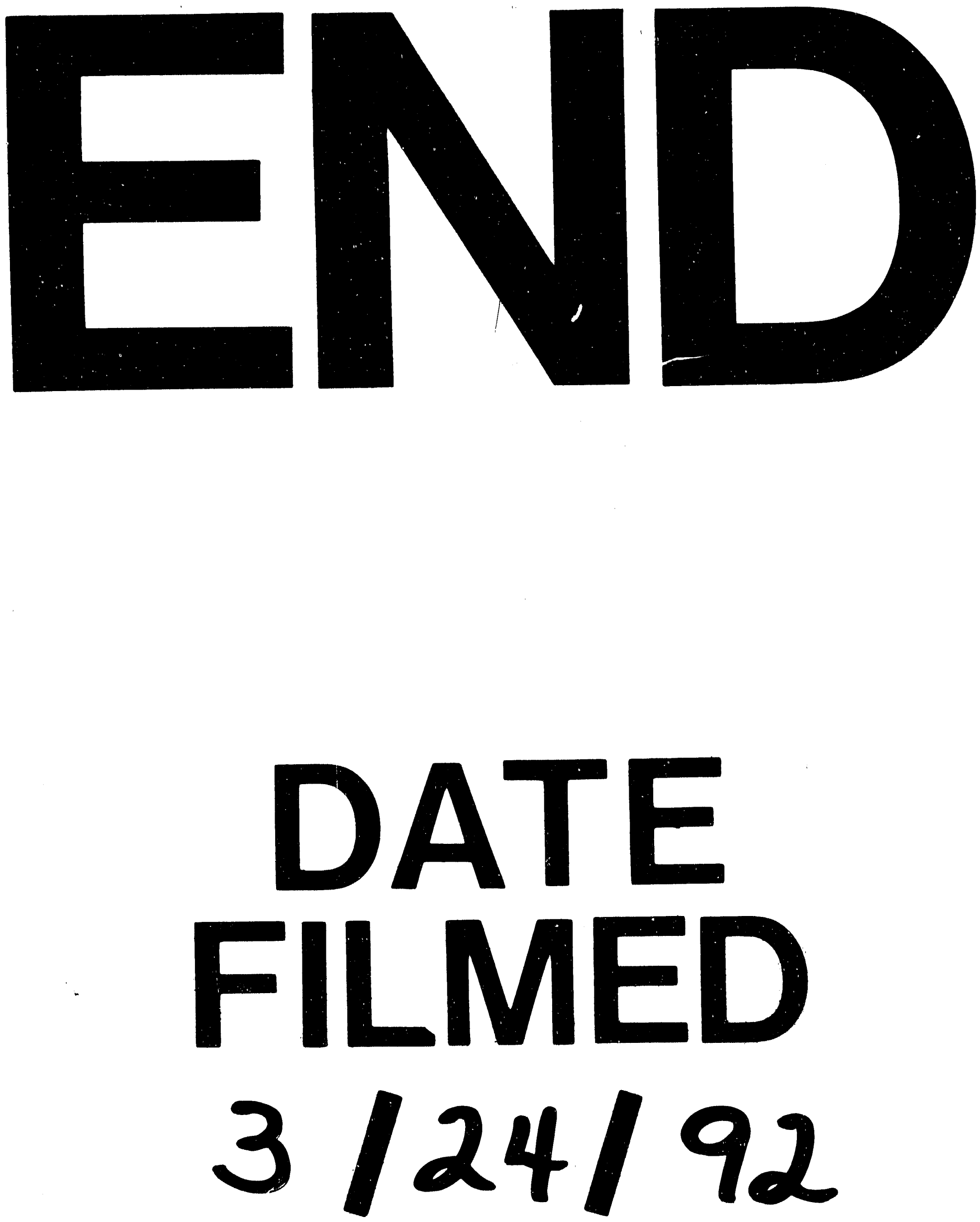
